\title{
A sustentabilidade econômico-financeira no Proesf em municípios do Amapá, Maranhão, Pará e Tocantins
}

\author{
The economic-financial sustainability \\ of the PROESF in the States of Amapá, \\ Maranhão, Pará and Tocantins
}

Ana Tereza da Silva Pereira 1

Ana Cecília Faveret de Sá Campelo 2

Fátima Scarparo Cunha ${ }^{3}$

José Noronha 4

Hésio Cordeiro 5

Sulamis Dain 6

Telma Ruth Pereira 7

\footnotetext{
1 Instituto de Medicina

Social e Fundação

Cesgranrio.

Rua Santa Alexandrina 1011,

Rio Comprido, 20261-235,

Rio de Janeiro RJ.

ana_pereira@terra.com.br

2 Agencia Nacional

de Saúde ANS.

3 Faculdade de

Enfermagem, UFRJ.

4 Fiocruz.

5 Fundação Cesgranrio.

6 Instituto de Medicina

Social, Uerj.

7 Fundação Cesgranrio.
}

\begin{abstract}
As part of the baseline studies of the PROESF (Project for the Expansion and Consolidation of Family Health), this article studies the funding of the PSF (Program for Family Health) from the viewpoint of its financial sustainability. It approaches aspects related to the construction of measures and indicators for sustainability based on a recent Brazilian political experience of differentiating the financial incentives given by the Unified Health System (SUS) through the PROESF to municipalities with more than 100 thousand inhabitants. The suggested inclusion of indicators only allows for defining the long-term coherence of the planned actions from the financial standpoint. This approach does not exclude the need for a critical review of the criteria currently used by the incentive policy of the PSF and its differentiation between municipalities, which is not exclusively based on their size. The differences in the situations of the municipalities with respect to sustainability pointed out in this study draw attention to the limits and limitations of applying alike policies to unalike situations, with foreseeable consequences of maintaining or worsening inequity.
\end{abstract}

Key words Primary health care, PSF, Financial incentives, Financial sustainability
Resumo Nos estudos de linha de base do Projeto de Expansão do Programa Saúde da Família (Proesf), o artigo trata do tema do financiamento do Programa de Saúde da Família (PSF) na ótica de sua sustentabilidade financeira. Aspectos relativos à construção de medidas e indicadores de sustentabilidade são abordados a partir da experiência recente de diferenciação da política de incentivos financeiros do SUS aos municípios de mais de 100 mil habitantes no Brasil através do Proesf. A sugestão de inclusão destes indicadores apenas permite definir a coerência a longo prazo das ações planejadas do ponto de vista de financiamento. O tema, entretanto, não esgota a necessidade de revisão crítica dos critérios hoje utilizados pela politica de incentivos do PSF e de sua diferenciação entre municípios, que não se deriva exclusivamente do porte municipal. As diferenças de situações municipais quanto à sustentabilidade, apontadas no estudo, chamam a atenção para os limites e limitações da aplicação de políticas homogêneas para situações desiguais, com conseqüências previsíveis sobre a manutenção e agravamento da iniqüidade.

Palavras-chave Atenção básica, PSF, Incentivos financeiros, Sustentabilidade financeira 


\section{Introdução}

Este artigo discorre sobre a formulação e aplicação de critérios para avaliação da sustentabilidade financeira do Programa de Saúde da Família (PSF), na sua inflexão, através do Projeto de Expansão do Programa Saúde da Família (Proesf) para a assistência à saúde nas grandes cidades, até então não alcançadas por este programa. Para tanto, analisará o financiamento público do setor saúde nos municípios dos Estados do Amapá, Maranhão, Pará e Tocantins, abrangidos pelos estudos de linha de base para avaliação do Proesf, em fase de realização pela Fundação Cesgranrio.

O Proesf tem como objetivo geral a realização de estudos de linha de base nos municípios selecionados, com vistas à institucionalização do monitoramento e avaliação das transformações decorrentes da conversão do modelo de atenção.

A par de apoiar a conversão de ações de saúde dos grandes centros ao modelo do PSF, o Proesf apóia iniciativas que têm por objetivo aprofundar as mudanças em curso nas localidades onde o programa já opera, integrando a diversidade de lógicas de atenção primária neles vigente, bem como ampliando a resolutividade do sistema de saúde como um todo.

Apesar da grande expectativa de que o PSF possa vir a ser a estratégia de reestruturação da atenção básica, e das mudanças recentes nas condições políticas e institucionais e de financiamento destinadas a acelerar este processo, poucas foram as cidades com sistemas de saúde complexos, onde o Programa foi introduzido como estratégia substitutiva do modelo assistencial. Na grande maioria das cidades de médio e grande porte, o PSF ainda é uma estratégia focalizada de expansão dos cuidados básicos de saúde, voltada a grupos populacionais sob maior risco social e expostos a precárias condições sanitárias.

Deve-se ressaltar que a mera adesão ao PSF ou a estratégias similares de atenção básica não garante, por si só, a dinâmica e o sucesso das ações voltadas à reorganização da rede como um todo, à melhoria das condições de gestão e ao aperfeiçoamento da gerência do Sistema Único de Saúde (SUS), sua expressão local.

$\mathrm{Na}$ perspectiva mais geral do financiamento, e com anterioridade à vinculação de recursos ao SUS e a programas e estratégias específicas, estudos gerais de Dain et al.1. destacavam as determinações do financiamento da Saúde decorrentes da estrutura e da reformulação do sistema tributário brasileiro, que abriu espaço para a maior autonomia municipal e para o difícil desafio de construção de um sistema único de saúde a partir da autonomia federativa no plano fiscal e das decisões de políticas públicas.

O mesmo estudo e também o estudo de Marques \& Mendes 2 examinam o financiamento da Saúde no contexto da construção e posterior repressão do Orçamento da Seguridade Social brasileira. Ambos os trabalhos apontam dificuldades ao financiamento da saúde em função da indevida apropriação de recursos deste orçamento, sinalizando para um quadro restritivo. A nosso juízo, este cenário torna mais relevante a afirmação de critérios adequados de incentivos em termos de financiamento à saúde, baseados na suficiência, na eqüidade e na sustentabilidade.

Entre os problemas centrais no âmbito do financiamento, destaca-se a questão dos incentivos financeiros ao PSF, com as conhecidas limitações dos critérios atuais de repasse federal aos municípios para o enfrentamento da questão do financiamento da atenção básica nas grandes cidades, bem como sua excessiva homogeneização.

No Brasil, em que pese o grande número de iniciativas voltadas para a consolidação do SUS e a existência de uma gama de projetos dirigidos para a avaliação das inovações que vem sendo introduzidas, não existe nenhuma iniciativa governamental no sentido de avaliar o impacto dessas mudanças no desempenho do sistema em seu conjunto.

A maioria das avaliações está voltada para o processo de descentralização ${ }^{3}$ ou de implantação de programas específicos, como o programa de Agentes Comunitários de Saúde (Pacs) e o Programa de Saúde da Família (PSF).

No campo do financiamento, estudos gerais vêm discutindo de forma agregada a questão das fontes e volume de recursos para o SUS, do ponto de vista de sua suficiência em relação à necessidade de expansão dos gastos de saúde ${ }^{1,3,4}$.

A necessidade de garantia de que os serviços e ações sejam realizados com qualidade e eficiência torna necessário o conhecimento, por parte do Estado, da repercussão financeira decorrente das transformações ensejadas pela extensão da cobertura e realização de todas as ações associadas ao PSF.

Existem poucos estudos de apuração de custos tendo em vista definir recursos como 
forma de financiamento de serviços associados ao PSF. Castro 5 trata com competência de aspectos ligados ao impacto financeiro de ações associadas aos custos da atenção básica.

Mais recentemente, a instituição do Sistema de Informações sobre Orçamentos Públicos em Saúde (Siops) trouxe novo alento para a análise para aspectos do financiamento e gastos de saúde no conjunto dos municípios brasileiros, como a feita por Faveret ${ }^{6}$ compatibili- $^{-}$ zando as informações e tornando-as parte do sistema de informações do SUS

Estudos avançam para a questão do caráter excessivamente monolítico dos incentivos federais. Neste sentido, Marques \& Mendes ${ }^{7}$ apontam para a eventual subordinação da autonomia estadual e municipal aos desígnios e prioridades do financiamento federal, o que pode ser constatado também nos casos analisados nesta pesquisa.

Melamed \& Rosário 8 analisam, neste contexto geral, as formas inovadoras de incentivo à atenção básica, destacando a importância dos incentivos estaduais, como formas adicionais e mais próximas às necessidades diferenciadas de recursos pelos municípios, decorrentes de suas condições específicas, destacando critérios adicionais utilizados para definição dos repasses.

Alternativas quanto à presença estadual na formulação de incentivos voltados para o tema da eqüidade na alocação de recursos para os municípios destacam-se na apresentação por Pelegrini \& Castro ${ }^{9}$ para o caso do Rio Grande do Sul, reforçando os pontos acima destacados, especificados para o programa de Saúde da Família por Senna10.

Também vêm sendo realizados estudos de caso que tratam da apreciação do impacto do PAB sobre estados e municípios selecionados, tais como os de Senna \& Cohen11; e Machado, Fortes e Somarriba 12 para o Estado de Minas Gerais. Na mesma direção, Marques \& Mendes ${ }^{13}$ apreciam criticamente a coerência da sistemática de incentivos dirigidos ao PSF.

Finalmente, Santos \& Gerschman 14 caracterizam de forma muito pertinente os determinantes institucionais, de oferta de serviços, e das relações entre provisão e produção de serviços que superam a mera questão da disponibilidade de recursos para o adequado equacionamento do tema do financiamento da atenção básica.

Embora cuidadosos e bem-sucedidos em seus propósitos específicos, tais estudos apóiamse em análises de impacto, não tratando da pro- jeção das situações evidenciadas para o médio e longo prazo.

A partir desta possibilidade, e já na perspectiva de ampliação do PSF para as grandes cidades brasileiras, foram realizados os primeiros estudos voltados ao desenvolvimento de um conceito de sustentabilidade financeira associado ao PSF15, a partir de estudos de caso realizados com a finalidade de apoiar o desenvolvimento do Proesf.

O presente estudo procura refinar a capacidade de avaliar e projetar a realidade financeira municipal diante dos desafios postos pela adesão à nova estratégia de transformação da atenção básica segundo o modelo do PSF.

Nele, a sustentabilidade é medida através da suficiência e permanência de recursos próprios e transferidos em relação às metas de cobertura pactuadas pelo Proesf. Os recursos mobilizados pelas finanças municipais, que definem o potencial de gasto per capita em saúde, são comparados com a projeção de despesas estimadas para os projetos de conversão.

Trata-se assim de buscar indicadores que avaliem se os municípios são capazes de fazer frente a gastos, direta ou indiretamente decorrentes do projeto a médio e longo prazo, associados ao processo de transformação da atenção básica.

Não há que se perder de vista, entretanto, que este é um estudo não finalizado, para o qual ainda concorrerão outras dimensões da pesquisa que enriquecerão a capacidade analítica realizada aqui apenas a partir de dados financeiros. Com esta limitação, é possível avançar resultados parciais que já sugerem a construção de indicadores financeiros capazes de subsidiar previsões sobre a maior ou menor estabilidade, em longo prazo, das experiências aqui contempladas.

\section{Elementos metodológicos para análise da sustentabilidade financeira em municípios selecionados}

Para descrever e analisar a situação econômico-financeira de 16 municípios selecionados para estudo no âmbito do Proesf, foram utilizados dados e indicadores do Sistema de Informações sobre Orçamentos Públicos em Saúde (Siops) e relatórios técnicos disponibilizados pela coordenação do projeto 12, 13, 14, 15. Foram realizados os procedimentos metodológicos descritos como se segue. 
Em primeiro lugar, foram feitos o levantamento, sistematização e análise dos dados e relatórios técnicos disponibilizados pela coordenação do projeto sobre a execução financeira do Proesf por parte do Ministério da Saúde e dos municípios.

Em seguida, foram realizados o levantamento, sistematização e análise de dados e dos indicadores econômico-financeiros do Siops sobre os municípios-alvo da pesquisa para a série 2002 a 2004.

O termo de referência do projeto que deu origem a este artigo sugere a inclusão, em estudos de linha de base, de indicadores que possam dimensionar a sustentabilidade econômico-financeira dos municípios do Proesf. Foi então feito o cálculo de indicadores de sustentabilidade econômico-financeira para os municípios constantes do estudo.

Os indicadores são dois, como segue:

\begin{tabular}{l}
\hline $\begin{array}{l}\text { Indicador } \mathbf{1} \\
\text { Despesa potencial em saúde per capita (DPSPC) }\end{array}$ \\
\hline DPSPC $=(0,15$ X RPPC $)+(0,15$ X TCPC $)+$ TSUSPC
\end{tabular}

Onde:

RPPC: Receita própria per capita

TCPC: Transferências constitucionais per capita

TSUSPC: Transferência SUS per capito

Indicador 2

Margem de expansão do gasto per capita (MEGPC)

MEGPC $=$ DPSPC - DAPC

Onde:

DPSPC: Despesa potencial em saúde per capita

DAPC: Despesa atual per capita

Com base nestes indicadores procedeu-se à elaboração de proposta de classificação dos municípios conforme os aspectos destacados de sustentabilidade econômico-financeira.

Finalmente, os resultados foram classificados de acordo com critérios especificados segundo sua execução financeira, evolução dos impostos diretamente arrecadados, cumprimento e a evolução dos resultados referentes à Emenda Constitucional 29.

\section{Classificação dos municípios segundo a execução financeira no período de 1 o/01/2003 a 31/12/2005}

- n.d.: informação não disponível ou não recebeu recursos do Ministério da Saúde

- Bom: executou mais de $90 \%$ dos recursos recebidos

- Razoável: executou entre $70 \%$ e $90 \%$ dos recursos recebidos

- Ruim: executou menos de $70 \%$ dos recursos recebidos

\section{Classificação segundo a evolução entre 2002 e 2004 do \% de impostos diretamente arrecadados}

- Positiva: diferença acima de $10 \%$

- Conservadora: diferenças entre $0 \%$ e $10 \%$

- Negativa: diferenças abaixo de $0 \%$

\section{Classificação segundo o cumprimento da EC 29 - Indicadores EC 29 (realizado e diferença em relação ao devido) de 2002 a 2004}

- Positiva: cumpriu a EC 29 em todos os anos estudados

- Negativa: descumpriu a EC 29 em pelo menos um dos anos estudados

\section{Classificação segundo a evolução do Indicador EC 29 de 2002 a 2004}

- Positiva: apresentou evolução acima de 15\%

- Estável: apresentou evolução entre 0\% e 15\%

- Negativa: apresentou evolução abaixo de $0 \%$

\section{Análise econômico-financeira dos 16 municípios}

Foram utilizados no trabalho dois tipos de relatórios: os produzidos pelos municípios que estão participando do Proesf e os produzidos pelo Ministério da Saúde. Os primeiros relatórios dizem respeito à caracterização do programa em cada município e à definição de metas físicas e financeiras para o período de 2003 (Ano 1) a 2008 (Ano 6). Os últimos são resumos do acompanhamento dos repasses feitos pelo Ministério aos municípios e da execução financeira por estes. As tabelas 1 e 2 sistematizam as informações contidas nos relatórios técnicos mencionados. 
Tabela 1

Resumo das metas financeiras anuais dos municípios - 2003 a 2008 .

\begin{tabular}{|c|c|c|c|c|c|c|c|}
\hline UF & Município & Ano 1 (2003) & Ano 2 (2004) & Ano 3 (2005) & Ano 4 (2006) & Ano 5 (2007) & Ano 6 (2008) \\
\hline AP & A & n.d & n.d & n.d & n.d & n.d & n.d \\
\hline \multirow[t]{6}{*}{ MA } & B & n.d & n.d & n.d & n.d & n.d & n.d \\
\hline & $\mathrm{C}$ & $205.730,23$ & $493.752,20$ & $329.168,39$ & & $329.168,39$ & $329.168,39$ \\
\hline & $\mathrm{D}$ & n.d & n.d & n.d & n.d & n.d & n.d \\
\hline & $\mathrm{E}$ & n.d & n.d & n.d & n.d & n.d & n.d \\
\hline & $\mathrm{F}$ & n.d & n.d & n.d & n.d & n.d & n.d \\
\hline & G & $82.118,00$ & $164.234,00$ & $295.621,50$ & $295.621,50$ & $197.080,50$ & $197.080,50$ \\
\hline \multirow[t]{7}{*}{ PA } & $\mathrm{H}$ & $129.336,80$ & $96.500,00$ & $345.854,00$ & $196.154,40$ & $137.169,50$ & $224.168,50$ \\
\hline & I & $623.442,00$ & $152.650,00$ & $826.615,00$ & $1.036 .006,80$ & $1.241 .748,00$ & - \\
\hline & $\mathrm{J}$ & $981.000,00$ & $751.000,00$ & $2.657 .000,00$ & $1.500 .000,00$ & $2.123 .000,00$ & $650.000,00$ \\
\hline & $\mathrm{L}$ & n.d & n.d & n.d & n.d & n.d & n.d \\
\hline & M & n.d & n.d & n.d & n.d & n.d & n.d \\
\hline & $\mathrm{N}$ & $85.000,00$ & $240.146,80$ & $505.000,00$ & $275.342,50$ & $385.000,00$ & $135.230,00$ \\
\hline & $\mathrm{O}$ & n.d & n.d & n.d & n.d & n.d & n.d \\
\hline \multirow[t]{2}{*}{ TO } & $\mathrm{P}$ & $121.000,00$ & $95.281,00$ & $307.574,00$ & $211.500,00$ & $286.000,00$ & - \\
\hline & Q & $811.908,00$ & $147.000,00$ & $1.333 .482,00$ & $623.140,00$ & $1.135 .050,00$ & $523.510,00$ \\
\hline
\end{tabular}

Fontes: Relatórios das Secretarias Municipais de Saúde. Elaboração própria.

Verifica-se, em primeiro lugar, a indisponibilidade de informações para alguns municípios. Em relação aos relatórios elaborados pelos próprios municípios, não constam os de metade dos 16 municípios, quais sejam: municípios A, B, D, E, L, M, O e o município F, cujo arquivo estava danificado. Este fato, por si só, compromete a análise consolidada dos dados, limitando sua comparabilidade.

A tabela 3 informa a situação do teto financeiro e da execução financeira dos municípios. Em todos os Estados, exceto Tocantins, os tetos dos municípios-alvo perfazem a maior parte do teto geral do Estado: 50,44\% no Amapá, 76\% no Maranhão, $81 \%$ no Pará e $45 \%$ no Tocantins, sendo as capitais responsáveis por mais da metade desses percentuais em cada Estado (coluna 1) Para dois municípios, $C$ e $M$ não houve repasse do Ministério da Saúde (coluna 2).

Em seis dos 15 municípios com informações de execução houve repasse de 100\% do teto previsto para a Fase 1: municípios D, E, F no Maranhão e município $H$ no Pará, além dos dois municípios do Tocantins. Para os outros sete municípios, os percentuais de repasse em relação ao teto variaram de $38,77 \%$ para o município A a 99,88\% para município O no Pará.

$\mathrm{Na}$ análise da execução financeira (coluna 3), três municípios ficam de fora: por não te- rem recebido repasses, e um, por não estar incluído nas planilhas do Ministério da Saúde. Dos 13 municípios com possibilidade de análise, destaca-se, negativamente, o município $\mathrm{O}$, que executou apenas $3,06 \%$ do repasse. Outros dois municípios ( $\mathrm{J}$ e Q) executaram menos de $50 \%$ do repasse.

Quatro municípios destacam-se positivamente por terem executado quase a totalidade dos recursos repassados - B /MA (99\%), F/MA (96\%), L/PA (92\%) e P/TO (99,9\%). Numa faixa aceitável estão os outros seis municípios: A/AP (71\%), D/MA (81\%), E/MA (88\%), G/MA (80\%),H/PA (62\%) e I/PA (86\%).

De modo resumido, em relação ao item “execução financeira” do projeto, poderíamos sugerir a seguinte classificação dos municípios:

\section{Dados e indicadores do Siops: \\ levantamento, sistematização e análise}

O Sistema de Informações sobre Orçamentos Públicos em Saúde (Siops) coleta, processa e disponibiliza para consulta pública dados sobre receitas dos municípios e sobre seus gastos com ações e serviços públicos de saúde.

Para a realização deste trabalho, o Siops foi utilizado para a coleta de dados e indicadores (automaticamente calculados) dos 16 municí- 
Tabela 2

Resumo da execução financeira de Estados e municípios (1@/01/2003 a 31/12/2005).

\begin{tabular}{|c|c|c|c|c|c|c|}
\hline UF & Município & Teto fase 1 & Repassado & Executado & $\begin{array}{l}\text { Saldo a } \\
\text { repassar }\end{array}$ & $\begin{array}{l}\% \text { Executado em } \\
\text { relação ao repasse }\end{array}$ \\
\hline \multirow[t]{3}{*}{ AP } & Geral & $1.112 .200,00$ & $282.500,00$ & $154.882,41$ & $829.700,00$ & 54,83 \\
\hline & Estado & $551.200,00$ & $65.000,00$ & - & $486.200,00$ & 0,00 \\
\hline & A & $561.100,00$ & $217.500,00$ & $154.882,41$ & $343.500,00$ & 71,21 \\
\hline \multirow[t]{8}{*}{ MA } & Geral & $3.334 .000,00$ & $2.272 .550,00$ & 2.007.441,07 & $1.061 .450,00$ & 88,33 \\
\hline & Estado & $798.000,00$ & $65.000,00$ & - & $733.000,00$ & 0,00 \\
\hline & B & $259.000,00$ & $152.600,00$ & $150.679,68$ & $104.600,00$ & 98,74 \\
\hline & $\mathrm{C}$ & $206.000,00$ & - & - & $206.000,00$ & n.a \\
\hline & $\mathrm{D}$ & $424.000,00$ & $424.000,00$ & $344.054,73$ & - & 81,14 \\
\hline & $\mathrm{E}$ & $212.000,00$ & $212.000,00$ & $186.075,01$ & - & 87,77 \\
\hline & $\mathrm{F}$ & $1.188 .000,00$ & $1.188 .000,00$ & $1.142 .639,97$ & - & 96,19 \\
\hline & G & $247.000,00$ & $230.950,00$ & $183.991,68$ & $16.050,00$ & 79,67 \\
\hline \multirow[t]{9}{*}{ PA } & Geral & $4.529 .200,00$ & $2.322 .000,00$ & $1.109 .277,47$ & $2.207 .200,00$ & 47,77 \\
\hline & Estado & $861.200,00$ & $65.000,00$ & - & $796.200,00$ & 0,00 \\
\hline & $\mathrm{H}$ & $226.000,00$ & $226.000,00$ & $139.101,00$ & - & 65,55 \\
\hline & I & $777.000,00$ & $623.500,00$ & $534.321,57$ & $153.500,00$ & 85,70 \\
\hline & $\mathrm{J}$ & $1.732 .000,00$ & $847.300,00$ & $355.094,40$ & $884.700,00$ & 41,91 \\
\hline & $\mathrm{L}$ & $184.000,00$ & $71.800,00$ & $65.800,00$ & $112.200,00$ & 91,64 \\
\hline & M & $260.000,00$ & - & - & $260.000,00$ & n.a \\
\hline & $\mathrm{N}$ & sem informação & sem informação & sem informação & sem informação & sem informação \\
\hline & $\mathrm{O}$ & $489.000,00$ & $488.400,00$ & $14.960,50$ & 600,00 & 3,06 \\
\hline \multirow[t]{4}{*}{ TO } & Geral & $1.145 .000,00$ & $578.000,00$ & $361.666,56$ & $567.000,00$ & 62,57 \\
\hline & Estado & $632.000,00$ & $65.000,00$ & - & $567.000,00$ & 0,00 \\
\hline & $\mathrm{P}$ & $217.000,00$ & $217.000,00$ & $216.923,00$ & - & 99,96 \\
\hline & Q & $296.000,00$ & $296.000,00$ & $144.743,56$ & - & 48,90 \\
\hline
\end{tabular}

Fontes: Relatórios do Proesf/Ministéro da Saúde. Elaboração própria.

pios em estudo, para a série histórica de 2002 a 2004. Com vistas a tornar sua utilização mais clara, classificamos tais dados e indicadores e formulamos a tabela 4 .

Após verificação de que todos os municípios haviam alimentado o Siops em todos os anos da série proposta, procedeu-se à efetiva coleta e sistematização dos dados. A partir da tabela completa, foram escolhidos dois indicadores para serem vistos com maior detalhe.

O primeiro foi a participação, em termos percentuais, da receita de impostos diretamente arrecadados, na receita total dos municípios. Trata-se de um indicador que pode ser utilizado para medir o grau de organização interna dos municípios para arrecadarem impostos e, de certo modo, a sua capacidade de gestão. Por outro lado, ele reflete a metodologia de cálculo das transferências intergovernamentais constitucionais e legais, em particular do Fundo de Participação dos Municípios. Verificou-se que as quatro capitais incluídas no estudo são os municípios com maior resultado nesse indicador.

Como proposta para nossa classificação, a tabela 5 sumariza o tipo de evolução do indicador estudado no período 2002 a 2004 para cada município, classificando como positivas as diferenças acima de $10 \%$, como conservadoras as diferenças entre $0 \%$ e $10 \%$, e como negativas as diferenças abaixo de $0 \%$.

O segundo indicador escolhido foi o de cumprimento da Constituição Federal no que tange ao percentual da receita destinado às despesas com ações e serviços públicos de saúde. O chamado "Indicador EC 29" foi tratado sob dois prismas - na constatação do cumprimento, ou não, da regra no período e na sua evolução. Note-se que este indicador aponta o esforço feito pelo governo municipal de aplicação de recursos próprios em saúde, sintetizando variáveis relativas à situação fiscal e financeira e à saúde propriamente dita. 


\begin{tabular}{|c|c|c|c|c|}
\hline UF & Município & $\begin{array}{l}\% \text { do teto do } \\
\text { município } \\
\text { no teto geral }\end{array}$ & $\begin{array}{l}\text { \% do repasse do } \\
\text { MS em relaçãoo } \\
\text { ao teto }\end{array}$ & $\begin{array}{l}\text { \% execução pelo } \\
\text { município do } \\
\text { repasse do MS }\end{array}$ \\
\hline AP & A & 50,44 & 38,77 & 71,21 \\
\hline \multirow[t]{6}{*}{ MA } & B & 7,77 & 58,92 & 98,74 \\
\hline & $\mathrm{C}$ & 6,18 & - & n.a \\
\hline & $\mathrm{D}$ & 12,72 & 100,00 & 81,14 \\
\hline & $\mathrm{E}$ & 6,36 & 100,00 & 87,77 \\
\hline & F & 35,63 & 100,00 & 96,18 \\
\hline & G & 7,41 & 93,50 & 79,67 \\
\hline \multirow[t]{7}{*}{ PA } & $\mathrm{H}$ & 4,99 & 100,00 & 61,55 \\
\hline & I & 17,16 & 80,24 & 85,70 \\
\hline & $\mathrm{J}$ & 38,24 & 48,92 & 41,91 \\
\hline & $\mathrm{L}$ & 4,06 & 39,02 & 91,64 \\
\hline & M & 5,74 & - & n.d \\
\hline & $\mathrm{N}$ & sem informação & sem informação & sem informação \\
\hline & $\mathrm{O}$ & 10,80 & 99,88 & 3,06 \\
\hline \multirow[t]{2}{*}{ TO } & $\mathrm{P}$ & 18,95 & 100,00 & 99,96 \\
\hline & Q & 25,85 & 100,00 & 48,90 \\
\hline
\end{tabular}

Fontes: Relatórios do Proesf/Ministério da Saúde. Elaboração própria

Quanto ao aspecto de cumprimento do texto constitucional, a tabela 6 demonstra os percentuais aplicados, a diferença em relação ao devido em pontos percentuais e se a aplicação devida foi efetuada ou não. Note-se que os percentuais devidos são de respectivamente 10,2\%, $11,8 \%$ e $15 \%$ para 2002, 2003 e 2004.

Na tabela 7, a evolução do indicador da EC29 serve de base para classificação municipal.

\section{Indicadores de sustentabilidade econômico-financeira}

A aplicação desses indicadores utilizando-se os dados e indicadores produzidos automaticamente pelo Siops requereu alguns procedimentos de adaptação e suscitou algumas reflexões.

Em primeiro lugar, foi necessário verificar quais os elementos da planilha do Siops já produzida com detalhamento dos dados e indicadores (Tabela 8) para todos os municípios e para a série de 2002 a 2004 deveriam ser utilizados. Para efeito de exemplificação e teste inicial, apresentamos a seguir os elementos identificados, para o município A, 2002 e a memória de cálculo dos indicadores de sustentabilidade.

Em seguida, adaptando as denominações dos indicadores àquelas utilizadas pelo Siops e aos dados e indicadores por ele produzidos automaticamente, chegamos ao detalhamento das fórmulas apresentadas na parte metodológica deste trabalho, a Despesa potencial em saúde per capita (DPSPC) e a Margem de expansão do gasto per capita (MEGPC).

Após o cálculo dos indicadores para a série histórica, permitimo-nos fazer algumas observações, quais sejam:

1) O Indicador 1 (despesa potencial em saúde per capita), ao considerar o fator de $15 \%$ para o cálculo da receita a ser aplicada em saúde, parece ter como referência o percentual de receita "vinculável" a ser gasto com ações e serviços públicos em saúde conforme a Constituição Federal (Emenda Constitucional no 29/00) a partir de 2004. Contudo, deve-se lembrar que os percentuais a serem aplicados em saúde conforme a EC 29/00 evoluíram da seguinte forma para o período em estudo: 2002 - 10,2\%; 2003 - 11,8\%; e $2004-15 \%$.

2) Os indicadores 1 e 2 (margem de expansão do gasto per capita), quando aplicados a um município, consideram as transferências intergovernamentais para a saúde como fixas.

Assim, para efeito de uma possível comparação, também calculamos o Indicador 1, com 
Tabela 4

Lista de dados e indicadores do Siops.

\begin{tabular}{|c|c|}
\hline $\begin{array}{l}\text { Tipo } \\
\text { Geral }\end{array}$ & $\begin{array}{l}\text { Dados e indicadores } \\
\text { População }\end{array}$ \\
\hline Indicadores de receita (Geral e Saúde) & $\begin{array}{l}\text { - Participação \% da receita de impostos na receita total do município } \\
\text { - Participação \% das transferências intergovernamentais na receita total do município } \\
\text { - Participação \% das transferências da União para a saúde no total de recursos } \\
\text { transferidos para saúde no município } \\
\text { - Participação \% das transferências da União para a saúde no total das transferências } \\
\text { da União para o município } \\
\text { - Participação \% da receita de impostos e transferências constitucionais e legais } \\
\text { na receita total do município }\end{array}$ \\
\hline Indicadores per capita (Saúde) & $\begin{array}{l}\text { - Despesa total com saúde por habitante } \\
\text { - Despesa com saúde feita com receita própria do município por habitante } \\
\text { - Transferências intergovernamentais para a saúde por habitante }\end{array}$ \\
\hline Indicadores de perfil de gasto (Saúde) & $\begin{array}{l}\text { - Participação \% da despesa com pessoal na despesa total com saúde } \\
\text { - Participação \% da despesa com medicamento na despesa total com saúde } \\
\text { - Participação \% da despesa com serviços de terceiros - pessoa jurídica na despesa } \\
\text { total com saúde } \\
\text { - Participação \% da despesa com investimentos na despesa total com saúde }\end{array}$ \\
\hline Indicadores relativos à Receita (Saúde) & $\begin{array}{l}\text { - Participação \% das transferências para a saúde na despesa total do município } \\
\text { com saúde } \\
\text { - Indicador EC } 29 \text { - \% da receita própria aplicada na saúde conforme EC29/2000 }\end{array}$ \\
\hline $\begin{array}{l}\text { Alguns dados de Receita } \\
\text { e Despesa (Geral e Saúde) }\end{array}$ & $\begin{array}{l}\text { - } \text { Despesa total com saúde } \\
\text { - } \text { Despesa com saúde feita com recursos próprios do município } \\
\text { - } \text { Receita de transferências intergovernamentais para a saúde } \\
\text { - } \\
\text { - } \text { Respesa com pessoal } \\
\text { - Receita de impostos diretamente arrecadados } \\
\text { - Receita do ICMS }\end{array}$ \\
\hline
\end{tabular}

Fonte: http://siops.datasus.gov.br/, acesso em 27/12/05. Elaboração própria.

impacto no Indicador 2, levando em conta a evolução esperada pela EC 29/00.

A tabela 9 consolida os dois indicadores calculados das duas formas (original 15\% e com variação segundo a EC 29/00).

A tabela 9 fornece um panorama geral da situação da despesa com saúde e de sua relação com as receitas próprias (impostos diretamente arrecadados e transferências constitucionais e legais) e com as transferências intergovernamentais para a saúde nos municípios selecionados para o estudo. A aplicação dos indicadores de despesa potencial e de margem de expansão do gasto com saúde, ambos per capita, ambos sugeridos no termo de referência do projeto, é feita para os anos de 2002 a 2004 de duas formas - uma considerando a fórmula original e outra, para efeito de comparação, considerando a evolução dos percentuais de vinculação de receitas segundo a Emenda Constitucional no 29/00.

O indicador DPSPC (despesa potencial em saúde per capita), ao ser analisado separadamente em qualquer dos dois cenários, apenas demonstra a grande variação da despesa com saúde entre os municípios selecionados. No Cenário 1, considerando-se 2002, os valores máximos e mínimos encontram-se no Maranhão: R\$ 56,32 no município E e R\$ 182,54 no município F. Nesse cenário, o município $\mathrm{E}$ permanece com o menor DPSPC para toda a série, ao passo que, em 2004, o município Q (TO) ultrapassa o valor máximo de F, chegando a $\mathrm{R} \$ 238$, 95, o mesmo ocorrendo no Cená- 


\begin{tabular}{lcc}
\hline $\begin{array}{l}\text { Tabela } \mathbf{5} \\
\text { Sugestão de classificação segundo a evolução do \% de impostos diretamente arrecadados - 2002-2004. }\end{array}$ \\
\hline Município & Tipo de evolução & $\begin{array}{c}\text { Evolução \% dos impostos } \\
\text { diretamente arrecadados }\end{array}$ \\
\hline H/PA & Positiva & 57,86 \\
A/AP & Positiva & 46,06 \\
E/MA & Positiva & 29,34 \\
N/PA & Positiva & 22,16 \\
P/TO & Positiva & 12,34 \\
I/PA & Conservadora & 9,57 \\
J/PA & Conservadora & 7,99 \\
O/PA & Conservadora & 4,70 \\
F/MA & Conservadora & 4,11 \\
C/MA & Conservadora & 0,84 \\
B/MA & Negativa & $-7,31$ \\
L/PA & Negativa & $-13,98$ \\
M/PA & Negativa & $-15,19$ \\
Q/TO & Negativa & $-16,06$ \\
D/MA & Negativa & $-19,82$ \\
G/MA & Negativa & $-41,19$ \\
\hline
\end{tabular}

Fonte: http://siops.datasus.gov.br/, acesso em 27/12/05. Elaboração própria.

\begin{tabular}{|c|c|c|c|c|c|c|c|c|}
\hline \multicolumn{9}{|c|}{$\begin{array}{l}\text { Tabela } 6 \\
\text { Indicadores EC } 29 \text { (realizado e diferença em relação ao devido) e indicação } \\
\text { para a classificação dos municípios - } 2002 \text { a } 2004 \text {. }\end{array}$} \\
\hline \multirow[t]{2}{*}{ UF } & \multirow[t]{2}{*}{ Município } & \multicolumn{2}{|c|}{2002} & \multicolumn{2}{|c|}{2003} & \multicolumn{2}{|c|}{2004} & \multirow[t]{2}{*}{ Apresenta diferença } \\
\hline & & Aplicação & Dif. EC 29 & Aplicação & Dif. EC 29 & Aplicação & Dif. EC 29 & \\
\hline AP & A & 11,0 & 0,8 & 13,4 & 1,6 & 15,5 & 0,5 & Positiva \\
\hline \multirow[t]{6}{*}{ MA } & $\mathrm{B}$ & 10,4 & 0,2 & 25,4 & 13,6 & 18,2 & 3,2 & Positiva \\
\hline & $\mathrm{C}$ & 20,1 & 9,9 & 15,8 & 4,0 & 10,5 & $-4,5$ & Negativa (2004) \\
\hline & $\mathrm{D}$ & 15,4 & 5,2 & 11,8 & 0,0 & 12,7 & $-2,3$ & Negativa (2004) \\
\hline & $\mathrm{E}$ & 10,0 & $-0,2$ & 19,1 & 7,3 & 16,6 & 1,6 & Negativa (2002) \\
\hline & $\mathrm{F}$ & 11,9 & 1,7 & 15,8 & 4,0 & 15,3 & 0,3 & Positiva \\
\hline & G & 20,6 & 10,4 & 13,0 & 1,2 & 9,0 & $-6,0$ & Negativa (2004) \\
\hline \multirow[t]{7}{*}{ PA } & $\mathrm{H}$ & 10,7 & 0,5 & 12,2 & 0,4 & 15,2 & 0,2 & Positiva \\
\hline & $\mathrm{I}$ & 12,9 & 2,7 & 20,6 & 8,8 & 19,3 & 4,3 & Positiva \\
\hline & $\mathrm{J}$ & 12,9 & 2,7 & 16,0 & 4,2 & 15,8 & 0,8 & Positiva \\
\hline & $\mathrm{L}$ & 14,1 & 3,9 & 11,9 & 0,1 & 12,6 & $-2,4$ & Negativa (2004) \\
\hline & M & 11,9 & 1,7 & 23,2 & 11,4 & 15,5 & 0,5 & Positiva \\
\hline & $\mathrm{N}$ & 17,1 & 6,9 & 20,1 & 8,3 & 17,6 & 2,6 & Positiva \\
\hline & $\mathrm{O}$ & 11,8 & 1,6 & 15,6 & 3,8 & 16,1 & 1,1 & Positiva \\
\hline \multirow[t]{2}{*}{ TO } & $\mathrm{P}$ & 17,9 & 7,7 & 20,1 & 8,3 & 16,5 & 1,5 & Positiva \\
\hline & Q & 17,5 & 7,3 & 17,6 & 5,8 & 17,9 & 2,9 & Positiva \\
\hline
\end{tabular}

Fonte: http://siops.datasus.gov.br/, acesso em 27/12/05. Elaboração própria. 
Tabela 7

Evolução dos indicadores EC 29 - 2002 a 2004 - e indicação para a classificação dos municípios.

\begin{tabular}{lcc}
\hline Municípios & $\begin{array}{c}\text { Diferença (\%) entre o } \\
\text { realizado e o devido (EC 29) }\end{array}$ & $\begin{array}{c}\text { Situação da evolução } \\
\text { do indicador EC 29 }\end{array}$ \\
\hline B & 75,7 & Positiva \\
E & 66,0 & Positiva \\
I & 49,3 & Positiva \\
H & 41,8 & Positiva \\
A & 40,7 & Positiva \\
O & 37,3 & Positiva \\
M & 30,7 & Positiva \\
F & 28,5 & Positiva \\
J & 22,7 & Positiva \\
N & 3,0 & Estável \\
Q & 2,4 & Estável \\
L & $-7,3$ & Negativa \\
P & $-10,2$ & Negativa \\
D & $-17,3$ & Negativa \\
C & $-48,0$ & Negativa \\
G & $-56,3$ & Negativa \\
\hline
\end{tabular}

Fonte: http://siops.datasus.gov.br/, acesso em 27/12/05. Elaboração própria.

\section{Tabela 8}

Dados e indicadores utilizados no cálculo da sustentabilidade.

\begin{tabular}{lcc}
\hline Dados e Indicadores & Município A & Células/fórmula \\
\hline População & 306.580 & $\mathrm{C} 3$ \\
$\begin{array}{l}\text { Despesa total com saúde por habitante } \\
\text { Transferências intergovernamentais }\end{array}$ & 69,80 & $\mathrm{C} 10$ \\
para a saúde por habitante & 36,15 & $\mathrm{C} 12$ \\
$\begin{array}{l}\text { Receita de impostos e de transferências } \\
\text { constitucionais e legais }\end{array}$ & $93.870 .085,00$ & $\mathrm{C} 21$ \\
$\begin{array}{l}\text { Indicador 1: Despesa potencial em saúde } \\
\text { per capita (DPSPC) }\end{array}$ & $\mathbf{8 2 , 0 8}$ & $\mathbf{( 0 , 1 5} \mathbf{x}(\mathbf{C} 2 \mathbf{1} / \mathbf{C} 3))+\mathbf{C 1 2}$ \\
Indicador 2: Margem de expansão do gasto & $\mathbf{1 2 , 2 8}$ & $\mathbf{D P S C P}-\mathbf{C 1 0}$ \\
per capita (MEGPC) & &
\end{tabular}

Fonte: http://siops.datasus.gov.br/, acesso em 27/12/05. Elaboração própria.

rio 2, uma vez que a vinculação de receita é a mesma $(15 \%)$.

As diferenças dizem respeito a dois fatores que informam o indicador: as receitas próprias e as transferências feitas pelas outras esferas de governo, principalmente a federal, para a saúde. Quanto à diferença gerada pelas receitas próprias, deve-se considerar que os principais elementos de diferenciação são a receita de impostos diretamente arrecadados, os repasses do Fundo de Participação de Municípios (FPM) e de Imposto sobre Circulação de Mercadorias e Serviços (ICMS). Quanto às transferências da saúde, seguem lógica própria, normalmente da capacidade instalada, e dependem do papel do município no sistema estadual ou supramunicipal de saúde.

De fato, mais relevante para a nossa análise é verificarmos a situação do indicador 2 (margem de expansão do gasto per capita), que compara o valor apurado no indicador 1 com o gasto atual com saúde, ambos incluindo as transferências intergovernamentais para a saúde e considerando-as fixas. Para tanto, foram elaborados dois gráficos, correspondendo à evolução deste indicador nos dois cenários propostos.

$O$ indicador 2 pode ser lido de duas formas. Tomando-se a tabela 9, por um lado, os 
Tabela 9

Indicadores de sustentabilidade econômico-financeira dos municípios selecionados (dois cenários).

\begin{tabular}{|c|c|c|c|c|c|c|c|c|c|c|c|c|}
\hline \multirow{3}{*}{$\begin{array}{l}\text { Cenários } \\
\text { Estados/ } \\
\text { Municípios }\end{array}$} & \multicolumn{6}{|c|}{ 1. Considera $15 \%$ da receita vinculável } & \multicolumn{6}{|c|}{$\begin{array}{l}\text { 2. Considera a progressão (\%) da receita } \\
\text { vinculável conforme a EC } 29 / 00\end{array}$} \\
\hline & \multicolumn{3}{|c|}{$\begin{array}{l}\text { Despesa potencial } \\
\text { em saúde per capita } \\
\text { (DPSPC) }\end{array}$} & \multicolumn{3}{|c|}{$\begin{array}{c}\text { Margem de expansão } \\
\text { do gasto per capita } \\
\text { (MEGPC) }\end{array}$} & \multicolumn{3}{|c|}{$\begin{array}{l}\text { Despesa potencial } \\
\text { em saúde per capita } \\
\text { (DPSPC) }\end{array}$} & \multicolumn{3}{|c|}{$\begin{array}{c}\text { Margem de expansão } \\
\text { do gasto per capita } \\
\text { (MEGPC) }\end{array}$} \\
\hline & 2002 & 2003 & 2004 & 2002 & 2003 & 2004 & 2002 & 2003 & 2004 & 2002 & 2003 & 2004 \\
\hline $\mathrm{AP} / \mathrm{A}$ & 82,08 & 88,78 & 93,76 & 12,28 & 4,91 & $(1,68)$ & 67,38 & 78,75 & 93,76 & $(2,42)$ & $(5,12)$ & $(1,68)$ \\
\hline $\mathrm{MA} / \mathrm{B}$ & 133,43 & 137,84 & 149,88 & 7,38 & $(15,58)$ & $(4,79)$ & 125,80 & 133,03 & 149,88 & $(0,25)$ & $(20,39)$ & $(4,79)$ \\
\hline $\mathrm{MA} / / \mathrm{C}$ & 90,89 & 98,11 & 106,22 & $(5,88)$ & $(1,04)$ & 14,84 & 85,39 & 94,06 & 106,22 & $(11,38)$ & $(5,09)$ & 14,84 \\
\hline MA/D & 145,47 & 165,21 & 201,87 & $(0,80)$ & 6,96 & 15,35 & 135,95 & 158,23 & 201,87 & $(10,32)$ & $(0,02)$ & 15,35 \\
\hline $\mathrm{MA} / \mathrm{E}$ & 56,32 & 68,33 & 71,81 & 5,58 & $(4,29)$ & $(14,58)$ & 50,94 & 65,00 & 71,81 & 0,20 & $(7,62)$ & $(14,58)$ \\
\hline $\mathrm{MA} / \mathrm{F}$ & 182,54 & 187,96 & 227,44 & 12,34 & $(3,25)$ & $(1,34)$ & 163,49 & 174,87 & 227,44 & $(6,71)$ & $(16,34)$ & $(1,34)$ \\
\hline $\mathrm{MA} / \mathrm{G}$ & 79,63 & 103,73 & 147,67 & $(7,27)$ & 2,53 & 20,50 & 73,35 & 99,63 & 147,67 & $(13,55)$ & $(1,57)$ & 20,50 \\
\hline PA/H & 57,97 & 66,08 & 74,64 & 4,42 & 3,02 & $(0,23)$ & 53,02 & 62,61 & 74,64 & $(0,53)$ & $(0,45)$ & $(0,23)$ \\
\hline $\mathrm{PA} / \mathrm{I}$ & 71,17 & 78,21 & 90,03 & 2,62 & $(7,85)$ & $(6,55)$ & 65,06 & 73,73 & 90,03 & $(3,49)$ & $(12,33)$ & $(6,55)$ \\
\hline $\mathrm{PA} / \mathrm{J}$ & 147,35 & 161,96 & 198,98 & 5,84 & $(3,16)$ & 3,96 & 134,09 & 152,26 & 198,98 & $(7,42)$ & $(12,86)$ & 3,96 \\
\hline $\mathrm{PA} / \mathrm{L}$ & 76,11 & 80,38 & 85,15 & 1,04 & 3,51 & 2,93 & 70,85 & 76,77 & 85,15 & $(4,22)$ & $(0,10)$ & 2,93 \\
\hline $\mathrm{PA} / \mathrm{M}$ & 112,81 & 126,85 & 164,26 & 4,66 & $(12,90)$ & $(5,06)$ & 105,69 & 121,80 & 164,26 & $(2,46)$ & $(17,95)$ & $(5,06)$ \\
\hline $\mathrm{PA} / \mathrm{N}$ & 99,85 & 117,75 & 130,18 & $(4,97)$ & $(14,43)$ & $(8,64)$ & 88,39 & 108,62 & 130,18 & $(16,43)$ & $(23,56)$ & $(8,64)$ \\
\hline $\mathrm{PA} / \mathrm{O}$ & 90,56 & 95,31 & 108,80 & 5,37 & $(1,08)$ & $(3,48)$ & 82,62 & 89,53 & 108,80 & $(2,57)$ & $(6,86)$ & $(3,48)$ \\
\hline $\mathrm{TO} / \mathrm{P}$ & 64,29 & 77,74 & 116,26 & $(6,46)$ & $(14,50)$ & $(3,82)$ & 53,41 & 68,57 & 116,26 & $(17,34)$ & $(23,67)$ & $(3,82)$ \\
\hline TO/Q & 175,45 & 188,96 & 238,95 & $(19,61)$ & $(21,38)$ & $(18,08)$ & 137,47 & 163,04 & 238,95 & $(57,59)$ & $(47,30)$ & $(18,08)$ \\
\hline MAE & 56,32 & 68,33 & 71,81 & 5,58 & $(4,29)$ & $(14,58)$ & 50,94 & 65,00 & 71,81 & 0,20 & $(7,62)$ & $(14,58)$ \\
\hline
\end{tabular}

Fonte: http://siops.datasus.gov.br/, acesso em 27/12/05.

Notas: (1) Todos os indicadores utilizam a receita realizada e a despesa liquidada. Elaboração própria.

municípios mais bem posicionados são aqueles que têm menor margem de expansão de gastos, ou seja, que já gastam com saúde além de sua despesa potencial (indicador 1) - são aqueles, nos gráficos acima, com margem de expansão negativa.

Sob este prisma, o município Q no Tocantins estaria na melhor posição para todos os anos estudados e em ambos os cenários, seguido do município E no Maranhão que, com o menor DPSPC para toda a série, tem uma importante margem negativa, isto é, embora com gasto per capita baixo, gasta hoje mais do que o seu potencial, calculado das duas formas propostas. Considerando o ano de 2004, os seguintes municípios apresentam margem de expansão dos gastos com saúde negativa (do mais negativo para o menos negativo).

- TO/Q: (R\$18,08)

- MA/F: (R\$14,58)

- PA/N: $(\mathrm{R} \$ 8,64)$

- PA/I: (R\$6,55)

- PA/M: $(\mathrm{R} \$ 5,06)$

- $\mathrm{MA} / \mathrm{B}(\mathrm{R} \$ 4,79)$

- TO/P: $(\mathrm{R} \$ 3,82)$
- PA/O: $(\mathrm{R} \$ 3,48)$

- AP/A: $(\mathrm{R} \$ 1,68)$

- MA/F: (R\$1,34)

- $\mathrm{PA} / \mathrm{H}:(\mathrm{R} \$ 0,23)$

O segundo modo de se ler o indicador é o inverso do acima, ou seja, o município que tiver maior margem de expansão do gasto com saúde é o que mais poderá incorporar novas equipes do Programa Saúde da Família e fazer os investimentos necessários para sua implantação, porque ainda tem "margem" para fazêlo. Nesta visão, estariam em melhor posição os cinco municípios que ainda não gastam com saúde os $15 \%$ da receita própria constitucionalmente previstos, listados a seguir com as respectivas margens per capita:

- MA/G: R\$20,50

- MA/D: R\$15,35

- MA/C: R $\$ 14,84$

- PA/J: R\$3,96

- PA/L: R\$2,93

Qualquer que seja a opção do analista ao interpretar este indicador, verifica-se como ponto de partida a heterogeneidade dos municípios do ponto de vista econômico-finan- 
ceiro e as grandes diferenças nas suas possibilidades atuais e futuras de virem a assumir compromissos financeiros importantes em futuro próximo.

\section{Conclusões}

Os resultados alcançados, ainda que parciais, sugerem uma forma de classificação de desempenho econômico-financeiro dos municípios (Tabela 10). Com isso, pretende-se contribuir, além da avaliação, para direcionar ações futuras, que aumentem a capacidade do Ministério da Saúde e dos governos estaduais no sentido de ampliar a consistência e dar maior particularidade aos incentivos financeiros do Proesf.

Os indicadores sugeridos, formulados e aplicados para um conjunto de municípios beneficiados pelo Proesf não são, em si mesmos, capazes de definir a condição de suficiência de recursos disponíveis para os municípios, uma vez que isso depende das condições particulares de cada um.

Estas condições dizem respeito à oferta de serviços disponíveis que, como se sabe, não é homogênea no SUS, à inserção rural ou urbana, às peculiaridades regionais que implicam diferenças de custos entre regiões, à proximidade ou não da região metropolitana e a serviços disponíveis exclusivamente nas capitais.

Entretanto, tais indicadores podem ser analisados no interior de cada município e cotejados com seus planos de expansão de médio e longo prazo, assim como com seus projetos de investimento, e podem vir a constituir importante sinalizador da viabilidade das ações pretendidas no contexto do financiamento da expansão do PSF nas grandes cidades.

Este é apenas o primeiro passo para o exame de alternativas para o desenvolvimento de modelos de financiamento da Atenção Básica nos municípios de mais de 100 mil habitantes no Brasil. Embora se aceite que os municípios analisados tenham elementos em comum com todos os municípios brasileiros de maior porte, é também verdade que o sistema de incentivos deve levar em conta as diferenças entre municípios, derivadas de sua realidade socioambiental, étnica, geográfica e epidemiológica.

A adoção de incentivos caracterizados por sua linearidade não leva em conta o critério microrregional. A formulação mais específica de incentivos relativos à atenção básica pressupõe escolhas em relação à regionalização e a forma de gestão do sistema de saúde local.

Tabela 10

Sugestão de classificação dos municípios segundo a situação econômico-financeira.

\begin{tabular}{lllll}
\hline Município & $\begin{array}{l}\text { Status quanto à } \\
\text { execução financeira } \\
\text { do PROESF }\end{array}$ & $\begin{array}{l}\text { Tipo de evolução \% dos } \\
\text { impostos diretamente } \\
\text { arrecadados }\end{array}$ & $\begin{array}{l}\text { Diferenças no } \\
\text { Indicador EC 29 em } \\
\text { relação ao devido }\end{array}$ & $\begin{array}{l}\text { Situação da evolução } \\
\text { do indicador EC29 }\end{array}$ \\
\hline H & Ruim & Positiva & Positivas & Positiva \\
I & Razoável & Negativa & Positivas & Positiva \\
P & Bom & Conservadora & Negativa (2004) & Negativa \\
J & Ruim & Negativa & Negativa (2004) & Negativa \\
L & Bom & Positiva & Negativa (2002) & Positiva \\
M & Não recebeu & Conservadora & Positiva & Negativa \\
B & recursos do MS & Negativa & Negativa (2004) & Positiva \\
C & Bom & Positiva & Positiva & Positiva \\
D & Não recebeu & Positiva \\
A & recursos do MS & Conservadora & Positiva & Negativa \\
N & Razoável & Positiva & Positiva \\
Q & Razoável & Negativa & Negativa (2004) & Estável \\
O & Sem informação & Positiva \\
E & quanto ao teto & Negativa & Positiva & Negativa \\
F & Ruim & Positiva & Positiva & Estável \\
G & Ruim & Conservadora & Positiva & Positiva \\
\hline
\end{tabular}

Fonte: MS - Ministério da Saúde. Elaboração Própria 
Tais escolhas devem preceder ou, no mínimo, se desenvolver de forma concomitante à formulação dos mecanismos de financiamento que as viabilizam, o que não ocorre neste caso em que o diagnóstico das condições atuais da atenção básica, da média e alta complexidade e dos modelos de gestão ocorre de forma simultânea à realização deste estudo.

Assim, essas conclusões têm necessariamente caráter preliminar, o que não diminui a relevância da dimensão do financiamento aqui analisada.

\section{Colaboradores}

AT Pereira e TR Pereira realizaram a pesquisa de campo e selecionaram os resultados relevantes para este artigo, após sistematização feita por FS Cunha. S Dain e ACFS Campelo elaboraram os procedimentos metodológicos para análise dos dados do Siops e do Proesf visando à construção de indicadores de sustentabilidade financeira. H Cordeiro e J Noronha participaram da elaboração do artigo, tanto em termos de sua formulação inicial como da análise critica dos resultados.

\section{Referências}

1. Dain S, Faveret AC, Cavalcanti CE, Carvalho D, Quadros WL. Avaliação dos impactos de reforma tributária sobre o financiamento da saúde nos anos 90. In: Negri B, Di Giovanni G, organizadores. Brasil: Radiografia da Saúde. 1ạ ed., v. 1. Campinas: Unicamp; 2001. p. 233-88.

2. Marques RM, Mendes A. Os dilemas do financiamento do SUS no interior da seguridade social. Economia e Sociedade 2005; 14(1):159-75.

3. Vianna SM, Piola SF, Guerra AJ, Camargo SF. O financiamento da descentralização dos serviços de saúde: critérios para transferências de recursos federais para Estados e municípios. Brasília: Ipea; 1991. (Documento de Política/no 3).

4. Piolla SF, Vianna SM. Economia da saúde: conceito e contribuição para a gestão da saúde. Brasília: Ipea; 1995. (Documento de Política/no 149).

5. Castro JD. A utilização do sistema de custeio por absorção para avaliar os custos da atenção básica em saúde: reformulações e aprimoramentos metodológicos. Anais do VI Congresso Brasileiro de Saúde Coletiva, Salvador, 2000.

6. Faveret AC. Federalismo fiscal e descentralização no Brasil: o financiamento da política de saúde na década de 90 e início dos anos 2000 [tese]. Rio de Janeiro: Programa de Pós-Graduação em Saúde Coletiva, Instituto de Medicina Social, Uerj; 2000. 
7. Marques RM, Mendes A. A política de incentivos do Ministério da Saúde para a atenção básica: uma ameaça à autonomia dos gestores municipais e ao princípio da integralidade? Cad Saúde Pública 2002; 18(Supl):163-71.

8. Melamed C, Costa NR. Inovações no financiamento federal à Atenção Básica. Rev C S Col 2003; 8(2):393401.

9. Pelegrini MLM, Castro JD, Drachler ML. Eqüidade na alocação de recursos para a saúde: a experiência do Estado do Rio Grande do Sul, Brasil. Rev C S Col 2005; 10(2):275-86.

10. Senna MCM. Eqüidade e política de saúde: algumas reflexões sobre o Programa Saúde da Família. Cad Saúde Pública 2002; 18(Supl):203-11.

11. Modelo assistencial e estratégia saúde da família no nível local: análise de uma experiência. Rev C S Col 2002; 7(3):523-35.
12. Machado ENM, Fortes FBCTP, Somarriba M. Efeitos da introdução do PAB sobre a distribuição de recursos e a prestação de serviços: o caso de Minas Gerais. Rev C S Col 2004; 9(1):99-111.

13. Marques RM, Mendes A. Atenção básica e Programa de Saúde da Família (PSF): novos rumos para a política de saúde e seu financiamento? Rev C S Col 2003; 8(2):403-15.

14. Santos MAB, Gerschman. As segmentações da oferta de serviços de saúde no Brasil: arranjos institucionais, credores, pagadores e provedores. Rev C S Col 2004; 9(3):795-806.

15. Dain S, organizador, Favoreto C, Caetano R, Macedo L T, Mattos R, Viacava F et al. Análise de reestruturação dos modelos assistenciais de saúde em grandes cidades: padrões de custo e formas de financiamento. Brasília: Ministério da Saúde; 2002.

Artigo apresentado em 27/02/2006

Aprovado em 31/03/2006

Versão final apresentada em 17/04/2006 